\title{
AN ASYMPTOTIC SOLUTION FOR A PAIR OF FIRST ORDER EQUATIONS
}

\author{
S. D. CONTE AND W. C. SANGREN
}

1. Introduction. In the regular case the classical method of obtaining the eigenvalues and eigenfunctions of the equation

$$
\frac{d^{2} y}{d x^{2}}+[\lambda-q(x)] y=0
$$

under Sturmian boundary conditions involves the use of asymptotic expressions. In the singular cases of (1.1) when the range of $x$ is infinite or semi-infinite instead of finite, Titchmarsh [1] has shown that such asymptotic solutions are also necessary in obtaining spectral and expansion theorems by the methods of complex variables. The authors have been concerned with generalizing the results of Titchmarsh to the following pair of equations

$$
\frac{d u}{d x}-[\lambda a(x)+b(x)] v=0, \quad \frac{d v}{d x}+[\lambda c(x)+d(x)] u=0 .
$$

Interest in this system was aroused by a consideration of the Dirac relativistic wave equations for a particle in a central field, the wave equations for the one-dimensional case being of the form (1.2). The authors have shown in an as yet unpublished report [5] issued by the Oak Ridge National Laboratories that most of the results of Titchmarsh generalize to the system (1.2) for the singular case $[0 \leqq x<\infty]$ but with the restriction $a(x)=c(x)$. In order to remove this restriction it is necessary first to obtain an asymptotic solution to (1.2) for large $\lambda$ in the regular case. For the case $a(x)=c(x)=1$ over the finite interval $[0 \leqq x \leqq 1]$, Hurwitz [3] has obtained such an asymptotic solution as well as an expansion theorem for an arbitrary function pair. In this paper we generalize the results of Hurwitz by presenting an asymptotic solution to the system (1.2) for arbitrary functions $a(x), c(x)$ subject only to the restrictions given below.

2. Asymptotic solution for large $\lambda$. Consider the system (1.2) over the finite interval $[0 \leqq x \leqq 1]$ under the following conditions: $b(x)$ and $d(x)$ are real functions of class $C^{\prime}$ over this interval, $a(x)$ and $c(x)$ are positive, nonvanishing functions of class $C^{\prime \prime}$, and $\lambda$ is a parameter, real or complex. By a solution of (1.2) is meant a pair of functions

Received by the editors February 1, 1953. 
$[u(x, \lambda), v(x, \lambda)]$ with continuous first derivatives which satisfies (1.2). Let the initial conditions be

$$
u(0)=k_{0} \alpha, \quad v(0)=k_{0}^{-1} \beta,
$$

where

$$
k(x)=[a(x) / c(x)]^{1 / 4},
$$

$k_{0}=k(0)$, and $\alpha, \beta$ are arbitrary real constants. We may then prove the theorem.

TheOREM I. The system (1.2), (2.1) has the following asymptotic solution for large $\lambda$

$$
u(x)=k(x)[\alpha \cos \xi+\beta \sin \xi]+O(1 / \lambda),
$$

$$
v(x)=k^{-1}(x)[-\alpha \sin \xi+\beta \cos \xi]+O(1 / \lambda),
$$

where

$$
\xi(x)=\lambda h(x)+g(x), \quad h(x)=\int_{0}^{x}(a c)^{1 / 2} d y,
$$

$$
g(x)=\frac{1}{2} \int_{0}^{x} \frac{b c+a d}{(a c)^{1 / 2}} d y .
$$

This solution is unique.

To prove this theorem let

$$
\begin{aligned}
& u(x)=U(x)+k(x)[\alpha \cos \xi+\beta \sin \xi]+\frac{L_{1}(x) \sin \xi}{\lambda} \\
& v(x)=V(x)+k^{-1}(x)[-\alpha \sin \xi+\beta \cos \xi]+\frac{L_{2}(x) \sin \xi}{\lambda},
\end{aligned}
$$

where

$$
\begin{aligned}
& L_{1}(x)=-\alpha k^{\prime}(x) /(a c)^{1 / 2}+\frac{\beta k(x)}{2}\left\{\frac{b}{a}-\frac{d}{c}\right\}, \\
& L_{2}(x)=\beta k^{\prime}(x) / a+\frac{\alpha k^{-1}(x)}{2}\left\{\frac{b}{a}-\frac{d}{c}\right\} .
\end{aligned}
$$

If we substitute (2.5) into (1.2) we obtain for $U, V$ the equations

$$
U^{\prime}-(\lambda a+b) V=P / \lambda, \quad V^{\prime}+(\lambda c+d) U=Q / \lambda
$$

where 


$$
\begin{aligned}
& P(x, \lambda)=\left(L_{1}^{\prime}-b L_{2}\right) \sin \xi+L_{1} g^{\prime} \cos \xi, \\
& Q(x, \lambda)=\left(L_{2}^{\prime}+d L_{1}\right) \sin \xi+L_{2} g^{\prime} \cos \xi .
\end{aligned}
$$

Now let $F=\left[F_{1}, F_{2}\right], G=\left[G_{1}, G_{2}\right]$ be solutions of the system

$$
y_{1}^{\prime}=-\lambda c y_{2}, \quad y_{2}^{\prime}=\lambda a y_{1},
$$

satisfying the boundary conditions

$$
\begin{array}{ll}
F_{1}(0)=1, & F_{2}(0)=0, \\
G_{1}(0)=0, & G_{2}(0)=1 .
\end{array}
$$

We define the pseudo-Wronskian of two solution pairs $F, G$ by $W(F, G)=F_{1} G_{2}-F_{2} G_{1}$. It is easy to show from (2.9) that this Wronskian is independent of $x$ and hence from condition (2.10) we have $W(F, G)=1$. If we multiply (2.7) first by $F_{1},-F_{2}$ respectively, add and integrate from 0 to $x$, then multiply by $-G_{1}, G_{2}$ and add and integrate, we obtain

$$
\begin{gathered}
F_{1} U-F_{2} V=\int_{0}^{x}\left(b F_{1} V+d F_{2} U\right) d y+\frac{R(x, \lambda)}{\lambda}, \\
-G_{1} U+G_{2} V=-\int_{0}^{x}\left(b G_{1} V+d G_{2} U\right) d y+\frac{S(x, \lambda)}{\lambda},
\end{gathered}
$$

where

$$
\begin{aligned}
& R(x, \lambda)=\int_{0}^{x}\left(P F_{1}-Q F_{2}\right) d y, \\
& S(x, \lambda)=\int_{0}^{x}\left(-P G_{1}+Q G_{2}\right) d y .
\end{aligned}
$$

These equations may be solved linearly for $U$ and $V$ to yield the following integral equations

$$
\begin{array}{r}
U(x, \lambda)=G_{2}(x, \lambda) \int_{0}^{x}\left(b F_{1} V+d F_{2} U\right) d y-F_{2}(x, \lambda) \\
\cdot \int_{0}^{x}\left(b G_{1} V+d G_{2} U\right) d y+\frac{N(x, \lambda)}{\lambda}, \\
V(x, \lambda)=-F_{1}(x, \lambda) \int_{0}^{x}\left(b G_{1} V+d G_{2} U\right) d y+G_{1}(x, \lambda) \\
\cdot \int_{0}^{x}\left(b F_{1} V+d F_{2} U\right) d y+\frac{M(x, \lambda)}{\lambda}
\end{array}
$$


where

$$
\begin{aligned}
& N(x, \lambda)=G_{2}(x, \lambda) S(x, \lambda)+F_{2}(x, \lambda) R(x, \lambda), \\
& M(x, \lambda)=F_{1}(x, \lambda) S(x, \lambda)+G_{1}(x, \lambda) R(x, \lambda) .
\end{aligned}
$$

Now the functions $F, G$ satisfy the system of equations (2.9) which can be written as the second order equation

$$
y_{1}^{\prime \prime}-\frac{c^{\prime}}{c} y_{1}^{\prime}+\lambda^{2} a c y_{1}=0,
$$

and under the transformation $\zeta(x)=\int_{0}^{x} c d s, X=y_{1}$, this becomes

$$
X^{\prime \prime}(\zeta)+\lambda^{2}[a / c] X(\zeta)=0
$$

Now it is known from the Sturm-Liouville theory [4] that the solutions of (2.16) on a finite interval as well as their first derivatives with respect to $\lambda$ are $O(1)$ for large $\lambda$ and bounded for all values of the independent variable on this interval. The same statement holds for the solutions $F$ and $G$ of (2.15) over the interval $0 \leqq x \leqq 1$. It is now evident from (2.8), (2.12), and (2.14) that for large $\lambda$

$$
\begin{aligned}
P(x, \lambda) & =O(1), & Q(x, \lambda) & =O(1), \\
R(x, \lambda) & =O(1), & S(x, \lambda) & =O(1), \\
N(x, \lambda) & =O(1), & M(x, \lambda) & =O(1) .
\end{aligned}
$$

The integral equations (2.13) can be written

$$
\begin{aligned}
& U(x, \lambda)=\frac{N(x, \lambda)}{\lambda}+\int_{0}^{x}\left[K_{11}(x, y) U(y)+K_{12}(x, y) V(y)\right] d y, \\
& V(x, \lambda)=\frac{M(x, \lambda)}{\lambda}+\int_{0}^{x}\left[K_{21}(x, y) U(y)+K_{22}(x, y) V(y)\right] d y,
\end{aligned}
$$

where

$$
\begin{aligned}
& K_{11}(x, y, \lambda)=d(y)\left[G_{2}(x, \lambda) F_{2}(y, \lambda)-F_{2}(x, \lambda) G_{2}(y, \lambda)\right], \\
& K_{12}(x, y, \lambda)=b(y)\left[G_{2}(x, \lambda) F_{1}(y, \lambda)-F_{2}(x, \lambda) G_{1}(y, \lambda)\right], \\
& K_{21}(x, y, \lambda)=d(y)\left[-F_{1}(x, \lambda) G_{2}(y, \lambda)+G_{1}(x, \lambda) F_{2}(y, \lambda)\right], \\
& K_{22}(x, y, \lambda)=b(y)\left[-F_{1}(x, \lambda) G_{1}(y, \lambda)+G_{1}(x, \lambda) F_{1}(y, \lambda)\right] .
\end{aligned}
$$

Since $F, G$ are bounded for all $x$ on $0 \leqq x \leqq 1$ and for large $\lambda$, it follows that

$$
\begin{array}{ll}
\left|K_{11}\right| \leqq K|d|, & \left|K_{21}\right| \leqq K|d|, \\
\left|K_{12}\right| \leqq K|b|, & \left|K_{22}\right| \leqq K|b|,
\end{array}
$$


where $K$ is the maximum value of the absolute values of the expressions in brackets in (2.18). On taking absolute values in (2.17) we have

$$
\begin{aligned}
& |U| \leqq \frac{\bar{M}}{|\lambda|}+K \int_{0}^{x}[|d| \cdot|U|+|b| \cdot|V|] d y, \\
& |V| \leqq \frac{\bar{M}}{|\lambda|}+K \int_{0}^{x}[|d| \cdot|U|+|b| \cdot|V|] d y,
\end{aligned}
$$

where $\bar{M}=\max [|N(x, \lambda)|,|M(x, \lambda)|]$.

We now require the following lemma.

LEMMA 1. Let $h_{1}, h_{2}$ be integrable on $0 \leqq x \leqq 1$; let $h_{1}, h_{2}, g_{1}, g_{2}$ be positive, with $g_{1}, g_{2}$ continuous, and let $C_{1}$ be constant. If

$$
\begin{array}{ll}
g_{1}(x) \leqq C_{1}+\int_{0}^{x}\left(h_{1} g_{1}+h_{2} g_{2}\right) d s, & 0 \leqq x \leqq 1, \\
g_{2}(x) \leqq C_{1}+\int_{0}^{x}\left(h_{1} g_{1}+h_{2} g_{2}\right) d s, &
\end{array}
$$

then

$$
\begin{aligned}
& g_{1}(x) \leqq C_{1} \exp \left\{\int_{0}^{x}\left(h_{1}+h_{2}\right) d s\right\}, \\
& g_{2}(x) \leqq C_{1} \exp \left\{\int_{0}^{x}\left(h_{1}+h_{2}\right) d s\right\}
\end{aligned}
$$

To prove the lemma rewrite the inequalities (2.20)

$$
g_{1}(x) \leqq C_{1}+y_{1}, \quad g_{2}(x) \leqq C_{1}+y_{1},
$$

where $y_{1}=\int_{0}^{x}\left(h_{1} g_{1}+h_{2} g_{2}\right) d x, d y_{1} / d x=\left[h_{1}(x) g_{1}(x)+h_{2}(x) \cdot g_{2}(x)\right]$. Multiplying by $h_{1}, h_{2}$ respectively and adding we get

$$
\begin{gathered}
h_{1} g_{1}+h_{2} g_{2}=\frac{d y_{1}}{d x} \leqq C_{1}\left(h_{1}+h_{2}\right)+y_{1}\left(h_{1}+h_{2}\right) \\
\frac{d}{d x}\left[y_{1} \exp \left\{-\int_{0}^{x}\left(h_{1}+h_{2}\right) d s\right\}\right] \\
\leqq C_{1}\left(h_{1}+h_{2}\right) \exp \left\{-\int_{0}^{x}\left(h_{1}+h_{2}\right) d s\right\} \\
=C_{1} \frac{d}{d x}\left[C_{2}-\exp \left\{-\int_{0}^{x}\left(h_{1}+h_{2}\right) d s\right\}\right] .
\end{gathered}
$$


Now integrate from 0 to $x$ to obtain

$$
y_{1}(x) \leqq C_{1}\left[\exp \left\{\int_{0}^{x}\left(h_{1}+h_{2}\right) d s\right\}-1\right]
$$

The substitution of this expression in (2.21) proves the lemma.

We now apply the lemma to (2.19), letting $C_{1}=\bar{M} /|\lambda|, h_{1}=K|d|$, $h_{2}=K|b|, g_{1}=|U|, g_{2}=|V|$. The conditions of Lemma 1 are obviously satisfied and hence

$$
\begin{aligned}
& |U| \leqq \frac{\bar{M}}{|\lambda|} \exp \left\{K \int_{0}^{x}[|d|+|b|] d s\right\} \\
& |V| \leqq \frac{\bar{M}}{|\lambda|} \exp \left\{K \int_{0}^{x}[|d|+|b|] d s\right\} .
\end{aligned}
$$

Since both $b$ and $d$ are integrable over $[0 \leqq x \leqq 1]$, it follows that $U=O(1 / \lambda), V=O(1 / \lambda)$ for $0 \leqq x \leqq 1$. From (2.5) it is now evident that $u(x), v(x)$ have the asymptotic solution specified in Theorem $\mathrm{I}$. This proves the theorem.

3. The expansion theorem. In 1926 Bliss [2] considered a system of $n$ first order equations involving a parameter $\lambda$ linearly, the coefficients in the system being real-valued and continuous over a finite interval. When the matrix of the coefficients satisfied another condition which he termed definite self-adjointness he was able to prove the existence of an infinite number of real characteristic values and further that an arbitrary function could be expanded into a series of the characteristic functions. In the proof he used methods analogous to those of the Hilbert-Schmidt integral equation theory. The system (1.2) is included in the general system treated by Bliss. For our case $n=2$, a new proof, yielding somewhat sharper results, of the existence of the eigenvalues and of an expansion theorem can be based on the asymptotic solution presented in this paper. The proof is omitted but the expansion theorem is given below:

Theorem II. Consider the system (1.2) under the Sturmian boundary conditions

$$
\alpha_{0} u(0)+\beta_{0} v(0)=0, \quad \alpha_{1} u(1)+\beta_{1} v(1)=0,
$$

where $\alpha_{0}, \beta_{0}, \alpha_{1}, \beta_{1}$ are real constants such that $\left|\alpha_{0}\right|+\left|\beta_{0}\right| \neq 0,\left|\alpha_{1}\right|$ $+\left|\beta_{1}\right| \neq 0$. The eigenvalues of the system (1.2), (3.1) are real, discrete, infinite in number and extend from $-\infty$ to $+\infty$. To each eigenvalue $\lambda_{n}$ there is a unique solution pair $\left[u_{n}, v_{n}\right]$. These solutions are orthonormal in the following sense 


$$
\int_{0}^{1}\left(c u_{m} u_{n}+a v_{m} v_{n}\right) d x= \begin{cases}0, & m \neq n \\ 1, & m=n\end{cases}
$$

If $f=\left[f_{1}(x), f_{2}(x)\right]$ is a function of class $C^{\prime \prime}$ satisfying the conditions (3.1), then the following expansions hold

$$
f_{1}(x)=\sum_{m=-\infty}^{\infty} c_{n} u_{n}(x), \quad f_{2}(x)=\sum_{n=-\infty}^{\infty} c_{n} v_{n}(x)
$$

where $c_{n}=\int_{0}^{1}\left(c f_{1} u+a f_{2} v\right) d x$. The convergence in (3.3) is uniform.

\section{BIBLIOGRAPHY}

1. E. C. Titchmarsh, Eigenfunction expansions, Oxford Press, 1946.

2. G. A. Bliss, $A$ boundary value problem for a system of ordinary linear differential equations of the first order, Trans. Amer. Math. Soc. vol. 28 (1926) p. $561 \mathrm{ff}$.

3. W. Hurwitz, An expansion theorem for a system of linear differential equations of the first order, Trans. Amer. Math. Soc. vol. 22 (1921) pp. 526-543.

4. E. L. Ince, Ordinary differential equations, Dover Publications, 1926, pp. 271273.

5. S. D. Conte and W. C. Sangren, An eigenfunction expansion associated with a pair of first order differential equations, Report, Oak Ridge National Laboratories, October 1952.

WAYNE UNIVERSITY AND

OAk Ridge National Laboratories 